University of Wollongong

Research Online

Australian Institute for Innovative Materials -

Papers

Australian Institute for Innovative Materials

$1-1-2019$

\title{
A direct 3D suspension near-field electrospinning technique for the fabrication of polymer nanoarrays
}

\author{
Alexander Nagle \\ University of Wollongong, arn734@uowmail.edu.au \\ Cormac Fay \\ University of Wollongong, cfay@uow.edu.au \\ Zhigang Xie \\ Deakin University \\ Gordon G. Wallace \\ University of Wollongong, gwallace@uow.edu.au \\ Xungai Wang \\ Deakin University
}

See next page for additional authors

Follow this and additional works at: https://ro.uow.edu.au/aiimpapers

Part of the Engineering Commons, and the Physical Sciences and Mathematics Commons

Research Online is the open access institutional repository for the University of Wollongong. For further information contact the UOW Library: research-pubs@uow.edu.au 


\title{
A direct 3D suspension near-field electrospinning technique for the fabrication of polymer nanoarrays
}

\author{
Abstract \\ Near-field electrospinning (NFES) is widely recognized as a versatile nanofabrication method, one \\ suitable for applications in tissue engineering. Rapid developments in this field have given rise to layered \\ nanofibrous scaffolds. However, this electrostatic fabrication process is limited by the electric field \\ inhibitory effects of polymer deposition. This leads to a major challenge: how to surpass this limitation on \\ planar/layered constructs. While the current focus in this area largely lies with the investigation of new \\ materials, techniques and increasing precision of NFES systems and patterning, exploration of complex \\ collector substrates is often restricted by (i) available technology and (ii) access to complex electrode \\ manufacturing tools. To achieve nanofiber arrays suspended in free space, this paper documents both the \\ development of an integrated NFES system and the potential of standing electrodes manufactured via \\ selective laser melting. This system was first tested by 2D patterning on planar silicon, using polyethylene \\ oxide polymer solution. To demonstrate suspension NFES, two patterns operating within and around the \\ standing electrodes produced high volume suspended nanoarrays. Image analysis of the arrays allowed \\ for the assessment of fiber directionality and isotropy. By scanning electron microscopy, it was found that \\ a mean fiber diameter of $310 \mathrm{~nm}$ of the arrays was achieved. Effectively manoeuvring between the \\ electrode pillars required a precision automated system (unavailable off-the-shelf), developed in-house. \\ This technique can be applied to the fabrication of nanofiber structures of sufficient volume for tissue \\ engineering. \\ Disciplines \\ Engineering | Physical Sciences and Mathematics

\section{Publication Details} \\ Nagle, A. R., Fay, C. D., Xie, Z., Wallace, G. G., Wang, X. \& Higgins, M. J. (2019). A direct 3D suspension \\ near-field electrospinning technique for the fabrication of polymer nanoarrays. Nanotechnology, 30 (19), \\ 195301-1-195301-10.
}

\section{Authors}

Alexander Nagle, Cormac Fay, Zhigang Xie, Gordon G. Wallace, Xungai Wang, and Michael J. Higgins 


\section{A Direct 3D Suspension Near-Field Electrospinning Technique for the Fabrication of Polymer Nanoarrays.}

Alexander R. Nagle ${ }^{1 \dagger}$, Cormac D. Fay ${ }^{1 \dagger}$, Zhigang $\mathrm{Xie}^{2}$, Gordon G. Wallace ${ }^{1}$, Xungai Wang $2^{*}$, and Michael J. Higgins ${ }^{1^{*}}$

1 ARC Centre of Excellence for Electromaterials Science, University of Wollongong, Innovation Campus, AllM Facility, Squires Way, North Wollongong, New South Wales 2500, Australia.

${ }^{2}$ Institute for Frontier Materials, Deakin University, Geelong, Victoria 3216, Australia.

${ }^{*}$ Corresponding Authors

${ }^{+}$Contributed equally to the study

Emails:

xungai.wang@deakin.edu.au (Professor X. Wang*)

mhiggins@uow.edu.au (Associate Professor M. J. Higgins*)

arn734@uowmail.edu.au (A. R. Nagle)

cfay@uow.edu.au (C. D. Fay)

a.xie1976@gmail.com (Z.Xie)

gwallace@uow.edu.au (G. G. Wallace) 


\begin{abstract}
Near-Field Electrospinning (NFES) is widely recognized as a versatile nanofabrication method, one suitable for applications in tissue engineering. Rapid developments in this field have given rise to layered nanofibrous scaffolds. However, this electrostatic fabrication process is limited by the electric field inhibitory effects of polymer deposition. This leads to a major challenge: how to surpass this limitation on planar/layered constructs. While the current focus in this area largely lies with the investigation of new materials, techniques and increasing precision of NFES systems and patterning, exploration of complex collector substrates is often restricted by (i) available technology and (ii) access to complex electrode manufacturing tools. To achieve nanofiber arrays suspended in free space, this paper documents both the development of an integrated NFES system and the potential of standing electrodes manufactured via Selective Laser Melting (SLM). This system was first tested by 2D patterning on planar silicon, using polyethylene oxide (PEO) polymer solution. To demonstrate suspension NFES, two patterns operating within and around the standing electrodes produced high volume suspended nanoarrays. Image analysis of the arrays allowed for the assessment of fiber directionality and isotropy. By SEM it was found that a mean fiber diameter of $310 \mathrm{~nm}$ of the arrays was achieved. Effectively manoeuvring between the electrode pillars required a precision automated system (unavailable off-the-shelf), developed in-house. This technique can be applied to the fabrication of nanofiber structures of sufficient volume for tissue engineering.
\end{abstract}

Keywords: electrospinning, suspension, near-field, nanofiber, electrode, nanoarray 


\section{Introduction}

Tissue engineering (TE) applies synthetic and biomaterials to the pursuit of reciprocating natural cellular environments. To achieve this, TE employs additive manufacturing processes to produce, supplement and support microtissue constructs. Native extracellular matrices are composed of nanofibrous protein networks, which provide cells with mechanical, structural and signalling cues, acting as a substrate for cell adhesion and growth. To imitate this matrix, fabrication processes must operate at all levels of resolution, from the macro to the nanoscale.

Following research showing the advantages of ultrafine fibers in cell viability and behavioural studies, nanofabrication has received growing attention for use in TE. Cells are capable of sensing individual polymer fibers with diameters as low as several nanometers ${ }^{1}$, while sub-micron diameter fibers have exhibited improved cytocompatibility ${ }^{2,3}$. Of the current nanofiber fabrication techniques, solution electrospinning is one of the most extensively applied due to its ease of use, low-cost set-up, variety of material choice, and its ability to deposit at room temperature ${ }^{4-6}$.

In the last decade, electrospinning techniques have been developed that allow for the controlled patterning of nanofibers. Reducing the working distance, as well as the applied voltage, prevents the jetting instability inherent to conventional electrospinning. In this Near-Field Electrospinning (NFES) regime, accurate motion of a planar electrode relative to the emitting tip allows for the control of fiber placement and morphology ${ }^{7,8}$. Recently, NFES has transitioned from writing 2D patterns to producing 3D ordered structures by direct layer-by-layer deposition ${ }^{9-14}$. This has greatly extended electrospinning capabilities, which has allowed researchers to develop 3D microfiber scaffolds for cell culture research ${ }^{15-18}$.

Despite the success of this layer-by-layer NFES process, there are inherent limitations to this technique. Insulating polymer suppresses the electric field, inhibiting deposition and limiting the number of layers achievable. This is problematic when considering that an ideal in vitro 3D culture working volume is on the order of $0.1 \mathrm{~cm}^{3} 16,19$. One method to resolve this volume limitation has been achieved by Visser et al. ${ }^{16}$, i.e. by delaminating and stacking two $1 \mathrm{~mm}$ high microfibrous scaffolds, generating an assembly sufficiently large enough for 3D cell culture. However, this method created microfibers, which is impractical for delicate or complex non-planar structures, in addition to being difficult to delaminate and assemble without fragmentation.

An alternative approach is necessary to reach high volume nanofibrous constructs. Electrospun nanofibers can be suspended between electrodes, bridging macroscale distances. Razor blade electrodes ${ }^{20,21}$, standing electrode pin arrays ${ }^{22,23}$, among other novel electrode collection devices, guide the electrospun jet by electric field localisation. However, in previous research involving suspension electrospinning, the majority apply conventional working distances ${ }^{20,23-25}$, which result in the production of chaotically placed fibers. Those which apply a near-field regime achieve low volume structures ${ }^{22,25,26}$, which do not meet ideal microtissue construct volume considerations.

In this paper we discuss the development of a NFES system, designed to implement a novel suspension NFES technique, which directly suspends nanofibers interdigitally between pillar electrodes. This technique patterns across these electrodes to achieve nanofibrous arrays large enough for 3D cell culture. These electrode pillars direct the electrospun polymer jet, as well as support suspended 3D nanofiber structures within the interstitial space. Current commercial systems fail to meet precision standards necessary and remain generalised, lacking specific design elements and functionality to examine this concept. Thus it was necessary to develop a system in-house, as what is currently 
available does not allow for specific modular components, or have a high precision. To best achieve the accuracy required for this study, computerized automation methods were employed, using a number of incorporated automatic elements and software systems. Selective Laser Melting (SLM) manufacturing allowed for the tailored fabrication of the 3D electrodes. Using these electrodes we demonstrate the patterning of uniaxially aligned nanofibrous meshes. 


\section{Materials and Methods}

\subsection{Materials}

Polyethylene oxide (PEO) powder (Average Mv 200,000) was purchased from Sigma-Aldrich Co. (Sydney, Australia). PEO (14 wt\%) was mixed with water/ethanol (3:2) using a magnetic stirring bar for $1 \mathrm{hr}$ to reach homogeneity. Titanium Grade 5 (Ti 6Al-4V) powder with spherical particle size $<63$ $\mu \mathrm{m}$, was purchased from TLS Technik GmbH (Bitterfeld, Germany).

\subsection{NFES device}

\subsubsection{Instrumentation}

A custom NFES device was designed, developed and assembled for the requirements of this study. It consisted of a platform mounted on the X-Y stage, a high voltage supply, a syringe pump mounted on the Z-stage, a camera and an enclosure (see figure 1). Three linear motorised stages were sourced (ALSQ150B, Zaber), which met the desirable features for this study: a high unidirectional accuracy (15 $\mu \mathrm{m}$ ), a maximum speed of $280 \mathrm{~mm} \cdot \mathrm{s}^{-1}$, and the capacity for control via an RS232 serial interface. Figure 1 (A-I) presents the schematic of the instrument assembly. Two stages were overlaid perpendicular to each other, allowing for movement in the $\mathrm{X}$ and $\mathrm{Y}$ directions, with a tertiary Z-stage holding a syringe pump (UMP3, World Precision Instruments Inc.). Each stage had a step resolution of $0.5 \mu \mathrm{m}$ and a repeatability of $<2.5 \mu \mathrm{m}$.

On the $\mathrm{X}$ stage, an acrylic laser cut platform was attached to position the collecting electrodes. A syringe, with a blunt tipped stainless steel needle ( $25 \mathrm{G}, 0.25 \mathrm{~mm}$ inner diameter), was mounted on the programmable syringe pump. This pump drove the syringe plunger using a linear force threaded shaft with a step resolution of $3.2 \mu \mathrm{m}$. The needle was positioned and held in a vertical orientation by a small stainless steel drill chuck, located by positioning screws. The needle was connected to a positive bias high voltage power supply (ES30, Gamma HV), via crocodile clip connectors and conductive cabling. An automatic cleaning device (ACD) was positioned beneath the tip (see supplementary Section S1 and figure S1.).

An enclosure was built from heat formed laser cut transparent acrylic to house the system. A front hatch was built to allow for access to the system and separate the system from the outside environment for a more controlled operation. Dehumidified air was fed into the case from a compressor source through a pressure regulator (AR40-04H) passing into the case to a terminal pneumatic silencer. The air was fed into the closed case to reduce the humidity to approximately $19 \% \mathrm{RH}$, measured using a temperature and humidity data logger (XC0424). 


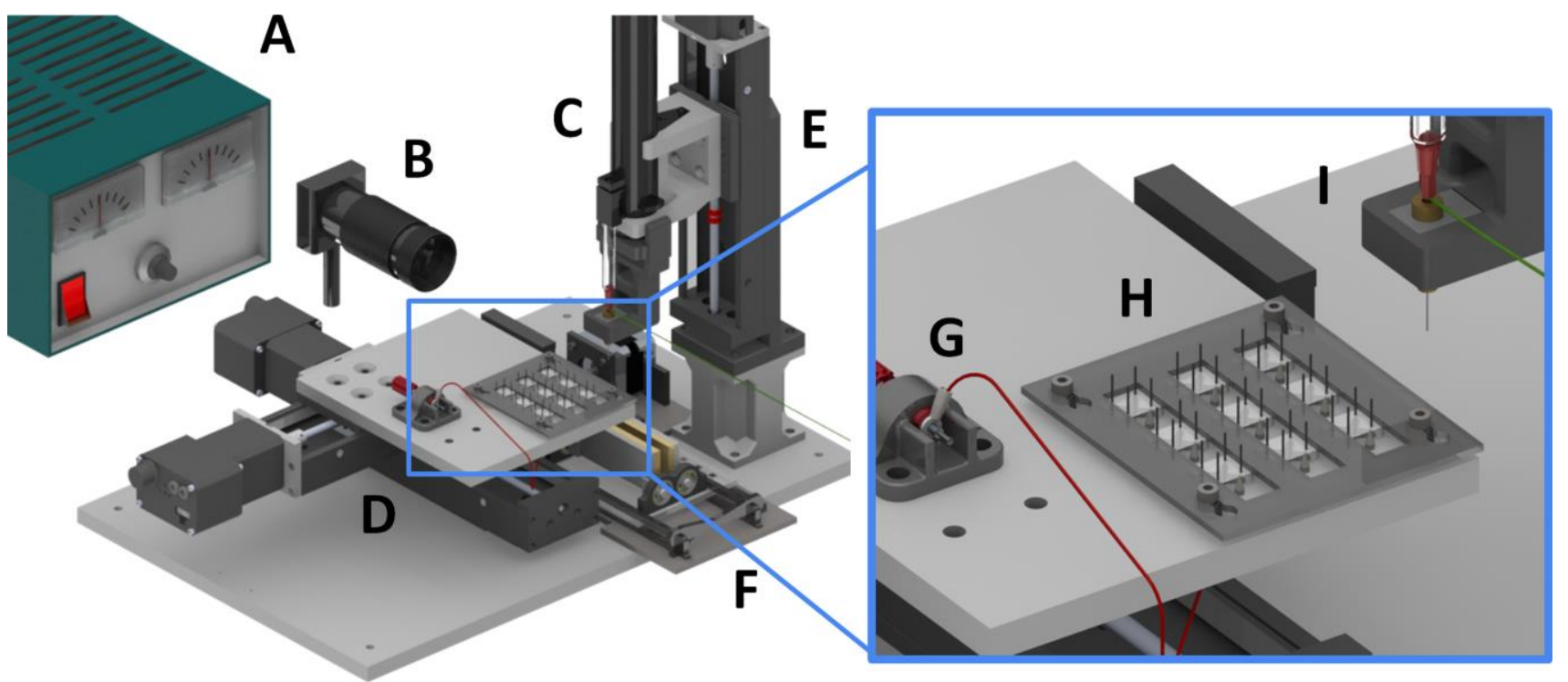

Figure 1. Design of the NFES system. (A) High Voltage Power Supply; (B) Camera; (C) Syringe pump; (D) X-Y Stage; (E) Z Stage; (F) Automatic Cleaning Device; (G) Positive bias contact point; (H) 3x3 grid array of electrodes embedded in the acrylic platform (I) Blunt tipped Needle, held by drill chuck, connected to ground.

\subsubsection{Software Design}

The stages, the syringe pump and the high voltage power supply were controlled by in-house developed software. For real-time control, along with desirable stability, the program was written in the C++ programming language using the Qt Framework (The Qt Company Ltd.). The graphical user interface (GUI) was composed of three rows; with the stages, syringe pump, and power supply controls arranged from top to bottom, respectively (see supplementary figure 2). Each row was separated into four compartments left to right: (1) variable control, (2) information (status), (3) action control (i.e. script), and (4) communications control, respectively.

The development of each peripheral control and sub-system took place in a progressive manner to ensure that all operations were successfully implemented via hard-coding. Once achieved, the GUI allowed for easy manual control of the individual instruments of the system. With all operations and features fully functional, automotive systems were subsequently developed. To allow for repeatable experiment operations and compatibility with 3D slicer programs, the automotive stage system was standardised operate using G-Code. This was achieved by implementing a script loading interface, parsing G-code input script and translating this to the native serial commands of the stages. This development enabled the user to carry out rapid 3D stage motion according to G-code program scripts.

G-code program scripts were output using an online spreadsheet program written in Google Sheets. Patterns were written by formatting the speed, $\mathrm{X}, \mathrm{Y}$ and $\mathrm{Z}$ values to $\mathrm{G}$-code format. Before each operation a series of calibrations were performed in order to mitigate error in positioning. First, a single pin electrode was moved beneath the emitting tip as a coordinate set point. Additional calibrations were performed for proceeding electrodes using a simple calibration script, which moves the pins of each electrode beneath the extrusion needle tip. Visual alignment took place using the magnified view via the camera. 


\subsection{Accuracy Validation}

\subsubsection{Planar NFES Dimensional Accuracy and Precision}

To evaluate the effective dimensional accuracy of the stage, in addition to comparing the conventional NFES patterning to the novel 3D patterning technique, 2D patterns of PEO polymer nanofibers were written on doped silicon wafers. The script translated the platform in two stages; (a) stabilisation, followed by (b) nine square wave patterns in a $3 \times 3$ grid. The stabilisation pattern consisted of $5 \mathrm{~mm}$ long parallel lines with an interspacing of $0.1 \mathrm{~mm}$. The square-wave patterns consisted of ten lines of $7 \mathrm{~mm}$ long parallel fibers, interspaced by $0.5 \mathrm{~mm}$. Each row of the $3 \times 3$ grid was assigned a stage maximum speed value: 50,150 , and $250 \mathrm{~mm} \cdot \mathrm{s}^{-1}$. Each $3 \times 3$ grid was repeated with varied working distances between $0.5-10 \mathrm{~mm}$ at an increment of $0.5 \mathrm{~mm}$. The following process variables were kept constant: the PEO solution concentration (14 wt\%), flow rate $\left(3 \mathrm{nl} . \mathrm{s}^{-1}\right)$, needle gauge $(0.25 \mathrm{~mm}$ inner diameter), and applied voltage $(1.6 \mathrm{kV})$.

PEO solution was loaded in a $1 \mathrm{ml}$ syringe and mounted on the syringe pump. A monochrome camera (Chameleon, PTGrey) was used to calibrate the relative Z-axis position of the blunt needle tip with respect to the electrode surface and to observe the ejection process. The needle tip was held at the $1 \mathrm{~mm}$ working distance and a droplet of the solution was formed at the outlet. The voltage was applied and the droplet was manually initiated by breaking the droplet surface using a fine metal tip. After several seconds the patterning was initiated. Upon pattern completion, the voltage was switched off and the needle was withdrawn. Each sample was reproduced in triplicate.

\subsection{Suspended Nanofiber Fabrication}

\subsubsection{Electrode fabrication and mounting}

Electrodes were produced using a SLM 3D Printer (SLM-50, Realiser) from grade 5 titanium powder. The electrodes were designed using 3D computer aided design (CAD) software (Solidworks 2016, Dassault Systèmes Solidworks Corp.) and consisted of four pins ( $0.7 \mathrm{~mm}$ diameter, $10 \mathrm{~mm}$ tall), standing vertically on an $\mathrm{x}$-shaped base, with two connection pins beneath the base (see figure $2 \mathrm{~A}$ ), which functioned to stabilise, position and connect the electrode. For SLM fabrication the design was saved in the stereolithography (.stl) file format. Using slicing software Magics (Materialise ${ }^{\circ}$ ), the file was sliced, and support structures were generated for printing. This sliced file was then interpreted into system specific commands by SLM proprietary software (R-Operator, Realiser). Grade 5 titanium powder was layered on the build platform. Each slice was welded by laser, with a layer resolution of approximately $25 \mu \mathrm{m}$. Once the electrodes were formed, the platform was removed and the electrodes cut from the surface using side cutters.

To mount the electrodes on the NFES stage, acrylic platforms were laser cut (PLS6MW, ULS inc.) with an exit opening to allow for ground connection to the pins from below. X-shaped grooves $(\sim 3 \mathrm{~mm}$ deep) were laser etched into the acrylic surface to allow for a press-fit insertion of the electrodes in to the platform. This mount was attached to the X-Y platform (by M3 screws) and the electrodes were securely fit within the formed grooves. Acrylic covers were attached over the base of the electrodes to electrically insulate the base from the syringe tip.

\subsubsection{Near-field electrospinning onto 3D electrodes}

A square wave (SW), inter-pin pattern was selected to generate ordered nanofibrous arrays in the region between two vertical pins. The working distance was defined as the minimum distance between the cylindrical surfaces of the tip and target pins. The upright pattern working plane was located at a certain working distance from two pins and parallel to the plane created by the pins (see figure $2 \mathrm{~B}$ ). Within the 
working plane the tip was rastered horizontally, raised with interspacings of $0.1 \mathrm{~mm}$ in the Z-axis. This formed the $7 \mathrm{~mm}$ wide SW pattern, with a total height of $5 \mathrm{~mm}$. There were two planes for each four pin electrode, parallel to one another (see supplementary video 1 for a demonstration of the process).

To calibrate the position of the syringe tip to the pins, the stage was moved to align one pin below the tip, and the tip was lowered until contact with the pin apex was achieved. A second calibration was performed to align the tip to the initiating pin. The G-code script was then adjusted by these calibrations. The SW pattern was preceded by an initiation procedure in which the solution droplet was contacted upon a fine point stainless steel tip. The SW pattern then followed its path as previously described. The initiation procedure disrupts the surface tension of the droplet allowing the emission process to commence more effectively.

A second pattern was selected to demonstrate the effective repeatability of the suspension NFES technique. This pattern was composed of a square path moving around all four pins in a clockwise fashion outside its perimeter at a minimum working distance of $1 \mathrm{~mm}$ (see figure $2 \mathrm{C}$ ). This clockwise path performed 15 laps at a single height and was then raised $0.1 \mathrm{~mm}$, and repeated this process. This sequence was repeated for a further nineteen $\mathrm{Z}$ axis positions, moving a total height of $1.9 \mathrm{~mm}$ (see supplementary video 2). This entire process was repeated a further nine times, effectively patterning at each position 150 times in total. This pattern demonstrates the systems can capably repeat the NFES technique in order to fabricate nanofibrous structures of comparatively high density.

This square iterative pattern (SI) was performed with preceding and interspersed cleaning manoeuvres in addition to initiation procedures. These procedures were necessary as the emission over time becomes destabilised due to surface tension, or contaminant particles disrupting the emission. Regular cleaning and initiation procedures mitigate these disruptions. The following process variables were kept constant for both patterns: the PEO solution concentration (14 wt \%), extrusion rate $\left(3 \mathrm{nl} . \mathrm{s}^{-1}\right)$, needle inner diameter $(0.25 \mathrm{~mm})$, maximum speed $\left(250 \mathrm{~mm} \cdot \mathrm{s}^{-1}\right)$ and working distance $(1 \mathrm{~mm})$.

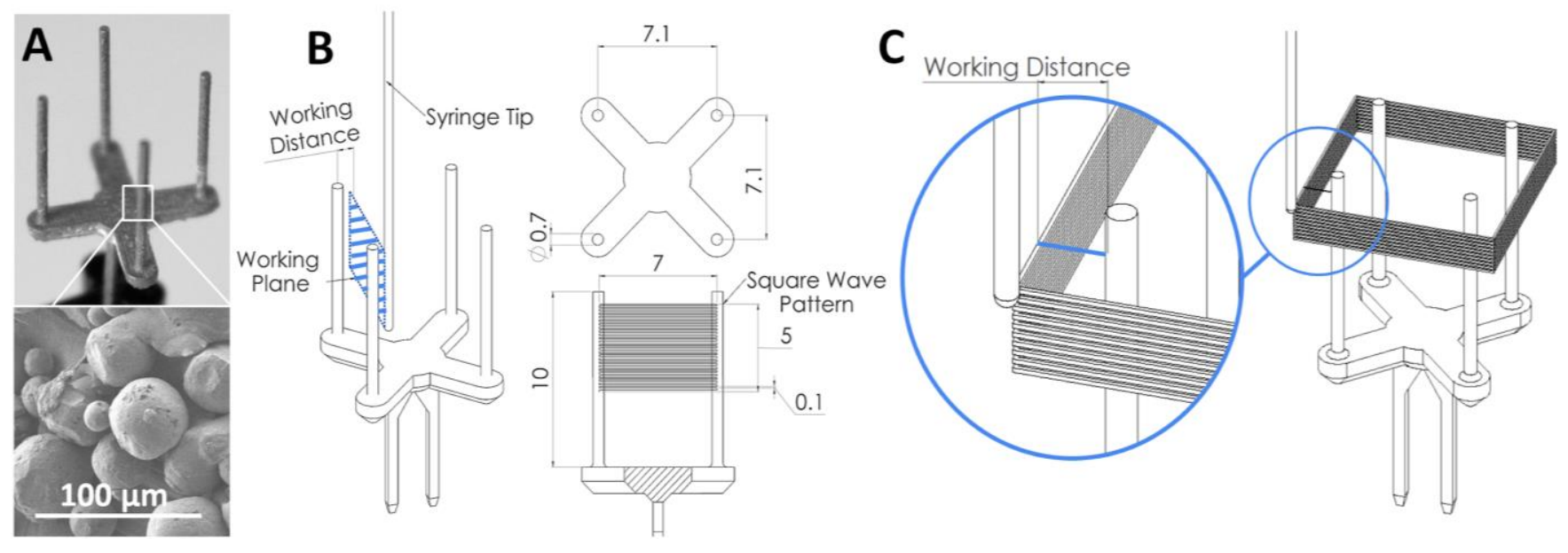

Figure 2. (A) Photograph of four pillar electrode with inset showing SEM of microparticle structure of semi-fused titanium. (B) Electrode schematic views (units in millimetres) showing a syringe tip movement working plane in blue; the dimensions of the electrode array, and the $0.1 \mathrm{~mm}$ interspaced square wave pattern dimensions between the two pins. (C) Pattern schematic of the square iteration (SI) pattern surrounding the four pin electrode, with magnified inset showing working distance. 


\subsection{Characterisation}

\subsubsection{Light Microscopy}

2D and 3D fiber arrays were imaged by light microscopy (M205A, Leica Microsystems). To achieve reproducible imaging of 3D suspended arrays the pins were held by their connection pins using bulldog clips mounted on 3D printed bases. Magnification was kept constant at $25 \times$ to enable comparative image analysis. Images of samples were semi-automatically analysed using Image J software (National Institutes of Health, USA). Images were first cropped then prepared using a variety of contrast enhancements and thresholding written as an automatic macro for ImageJ. The binary segmented images produced were then analysed using ImageJ plugin 'Diameter J', which applies the OrientationJ module to determine the orientation distribution (OD) of the fibers.

\subsubsection{Scanning Electron Microscopy (SEM)}

Prior to SEM imaging, silicon wafers and pin electrodes were attached to stainless steel stubs using carbon tape. Electrical contact between the electrodes and the base was ensured by using conductive silver paint. Note: the fibers were not coated with any conductive metal. This avoided the potentially destructive sputter coating process as well as allows SEM acquisition of fibers due to the higher contrast as a consequence of charge build up on fiber surface. SEM was performed using a JEOL 7500 operating at $10 \mathrm{kV}$, and $5 \mathrm{~mA}$. From the SEM micrographs the diameters of the fibers were measured. An excess of 10 measurements per SEM micrograph were performed, with a minimum of four micrographs per sample. From these measurements the average fiber diameter and standard deviation were calculated. 


\section{Results and Discussion}

\subsection{Accuracy Validation by Planar Nanofiber Patterning}

To evaluate 2D nanofiber patterning capabilities, a $3 \times 3$ grid of patterned lines was produced with three subgroups, varying maximum speed for each row. Bare planar silicon electrodes were chosen as a substrate to minimize surface artefact interference in both NFES patterning and imaging. The range of working distances was chosen given the typical working range for NFES is ca. $0.5-10 \mathrm{~mm}$, with previous findings showing an improved patterning precision working at the lower end of this range. From an examination of working distances between $0.5-10 \mathrm{~mm}$ (at an increment of $0.5 \mathrm{~mm}$ ); $6 \mathrm{~mm}$ to $8 \mathrm{~mm}$ were found to produce the most accurate patterns (see supplementary section figure S3). Arcing occurred at $0.5 \mathrm{~mm} \mathrm{WD}$, inhibiting stable emission, while working between $8.5-10 \mathrm{~mm}$ electrospinning could only be briefly maintained using $1.6 \mathrm{kV}$. No significant effect in patterning at the macroscale was found between the maximum speeds applied $(50,150$ and $250 \mathrm{~mm} / \mathrm{s})$. The reproducibility of these outcomes demonstrate the system's control over working events and conditions

Figure 3 A-C illustrates an increase in writing fidelity as the working distance is increased from 1-2 $\mathrm{mm}$. The jetting regime changes from multiple splaying jets $(1 \mathrm{~mm})$, to fewer jets $(1.5 \mathrm{~mm})$, to a single coherent jet $(2 \mathrm{~mm})$ as the working distance is increased. For working distance from 1.5 - $5 \mathrm{~mm}$ finite regions of inhomogeneous deposition to the left and right extremes of the arrays can be observed (see figure 3 (B-D)). This inhomogeneity is not due to the onset of electric bending instabilities, rather it is caused by the stage drawing the fiber away from the origin, causing angular deflection of the jet ${ }^{27,28}$. The force of drawing is lower on the jet, meaning its direction does not change as quickly as in $1 \mathrm{~mm}$ samples, leading to pooling of the jet at one point, due to the latency in drawing force.

Patterning accuracy becomes ideal between 5 and $6 \mathrm{~mm}$ (fig. 3 (D-E)) as a result of effective drying of the solution, which inhibits such fluid flow effects. A similar transition can be seen between 1 and $1.5 \mathrm{~mm}$, with multiple smaller jets drying at a faster rate, due to the increased surface area. Figure $3 \mathrm{~F}$, shows the normalised OD for the arrays (Fig. $3 \mathrm{~A}-\mathrm{E}$ ), with the inset showing a $80-100^{\circ}$ region visually demonstrating an ideal raster pattern for figure $3 \mathrm{~A}$ and $\mathrm{E}$.

It was practical to establish a 2D NFES technique using this platform in order to validate the platform writing operation, and to establish a working parameter domain as a starting point for 3D suspension NFES. Finally, this allowed a comparison to take place in order to explore differences between techniques, in terms of outcome and jetting behaviours. Parameter conditions are equivalent between the two techniques of 2D patterning and 3D suspension. The distinguishing characteristics between the techniques were defined by their respective substrates and applied patterns. In 2D NFES, working distance does not vary, producing a fixed electric field with respect to the emitter. In 3D NFES the angle of the jet is not aligned with the emitter, and the electric field varies as the electrodes move relative to one another. 


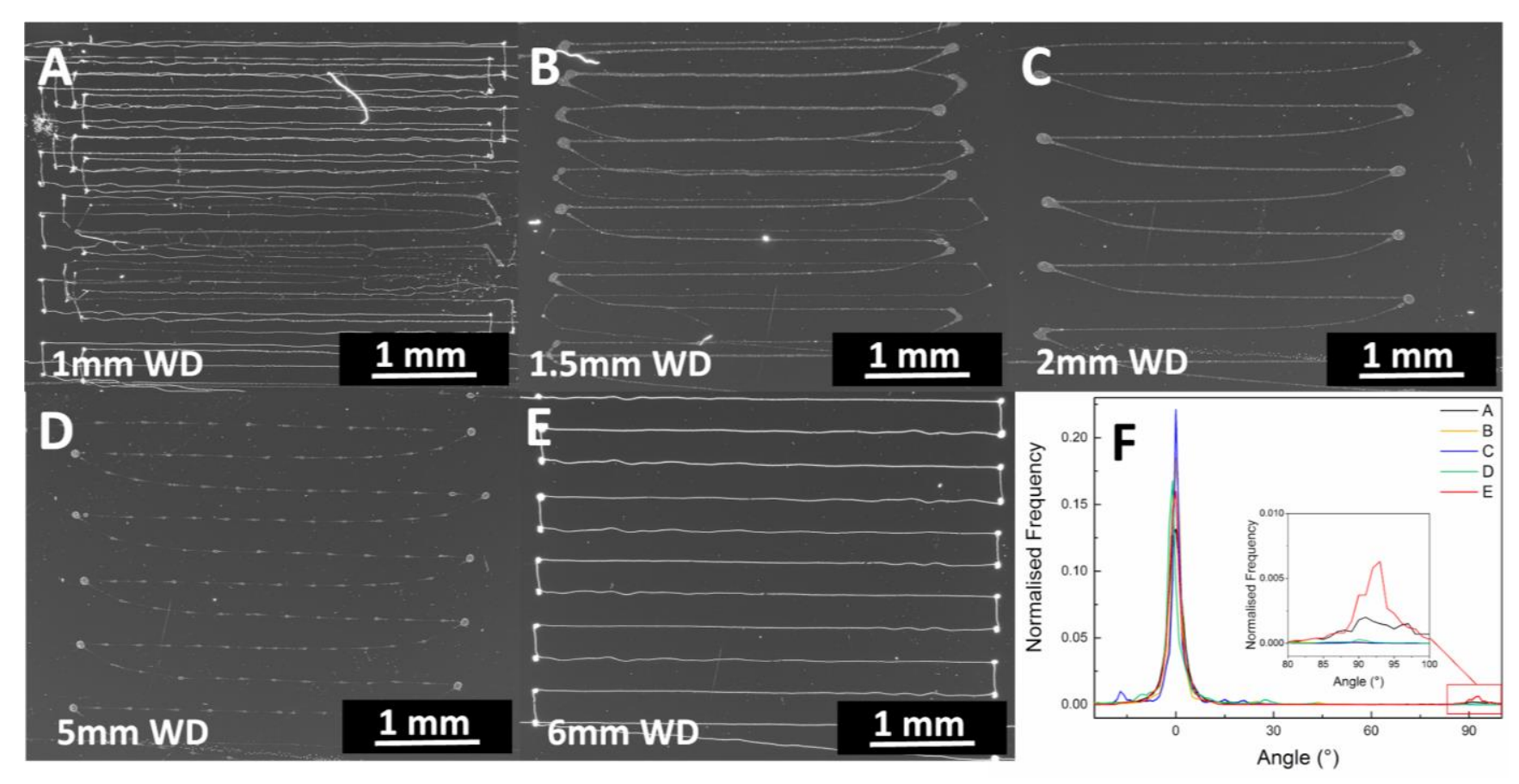

Figure 3. Planar Nanofiber Patterning. (A-E) Microscopy images of PEO fibers on silicon wafers at different working distances. (F) The OD for images A-E, with inset showing region of interest in the 80$100^{\circ}$ range.

\subsection{D Suspended Nanofiber Array Fabrication}

\subsubsection{Nanofiber Suspension by Square Wave pattern}

Two 3D patterns were demonstrated, SW and SI, producing suspended nanofibrous arrays upon 3D electrodes. The optimal product of the SW pattern is to generate homogenous axially aligned nanofibers, $90^{\circ}$ relative to the pins, and spaced by $0.1 \mathrm{~mm}$ over a height of $5 \mathrm{~mm}$. The arrays were characterised by these ideal standards in terms of orientation distribution, and fiber diameter.

Figure 4 (A) shows microscopy of the nanofibers spanning the region between pin electrodes. Fiber arrays were assessed for their order and this was quantified in terms of their normalised OD. Figure 4 (B) shows an SW array in the interspacing of electrodes, with a segmented overlay generated using ImageJ processing. The OD of this segment is shown in figure $4(\mathrm{C})$, with a centrepoint of approximately $90^{\circ}$ by Lorentz fitting, which in general showed improved fitting compared with Gaussian fitting. Bowing during SNFES can cause chaotic interactions with previously deposited fibers, resulting in entanglement as well as disruption to the continuing deposition. To achieve tautly suspended nanofibers, the move between pins must occur such that the fiber is not drawn beyond breaking point while minimizing the bowing of the fiber my maximising the speed of drawing. 


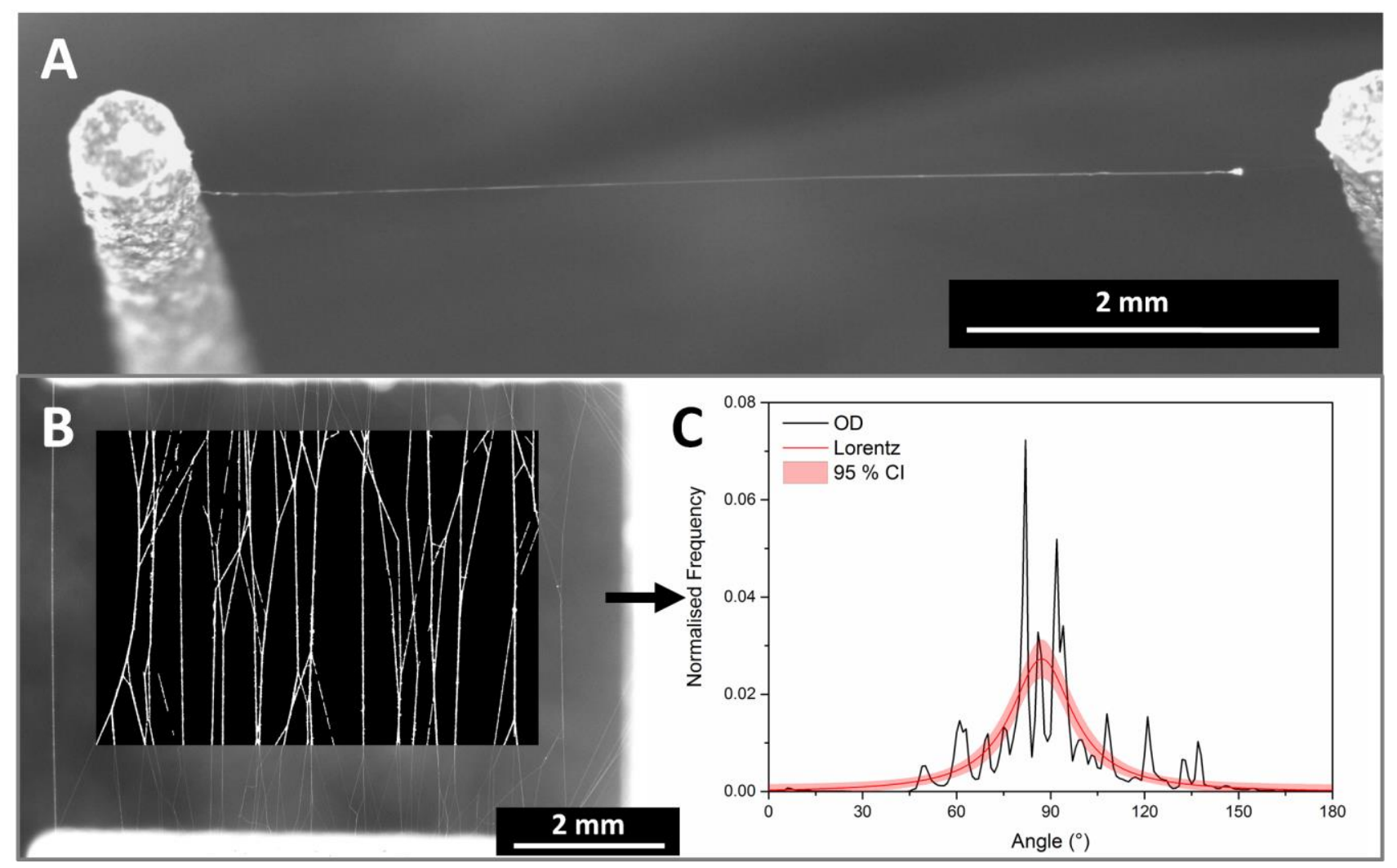

Figure 4. Microscopy and analysis of suspended nanofiber arrays. (A) Microscopy image of PEO fibers spanning the region between two electrodes (B) Fiber array with overlay showing a segmented view of the fibers. (C) The OD for image A, with Lorentz fitting centre point of $87.17^{\circ} \pm 0.83 \mathrm{SE}$.

\subsubsection{Nanofiber Suspension by Square Iteration Pattern}

A second pattern, SI, was applied to demonstrate the effectiveness of the automatic systems. Figure 5 (A) shows a photograph of the nanofibers on the electrode. Figure 5 (B) shows a top down microscope image of the suspended array at $25 \mathrm{x}$ magnification, illustrating the inter-pin fiber alignment. A higher magnification in Figure $5(\mathrm{C})$, shows the fibres tautly suspended, with polymer build-up at the corner of the pillar. The nanofibers spanning the electrodes from a side view as shown in figure 5 (D), and magnified in figure $5(\mathrm{E})$, exemplify the increase in fibre density compared with the SW patterning. This also shows the clear restriction of the disposition to approximately a $2 \mathrm{~mm}$ region, as determined by the SI pattern parameters.

The repeated SI pattern was the most simple to implement while demonstrating the automatic systems. It can be processed in any sequence as the toolpath never passes through the plane of deposited fibers (see supplementary video 3 for magnified observation of the SI pattern process). 


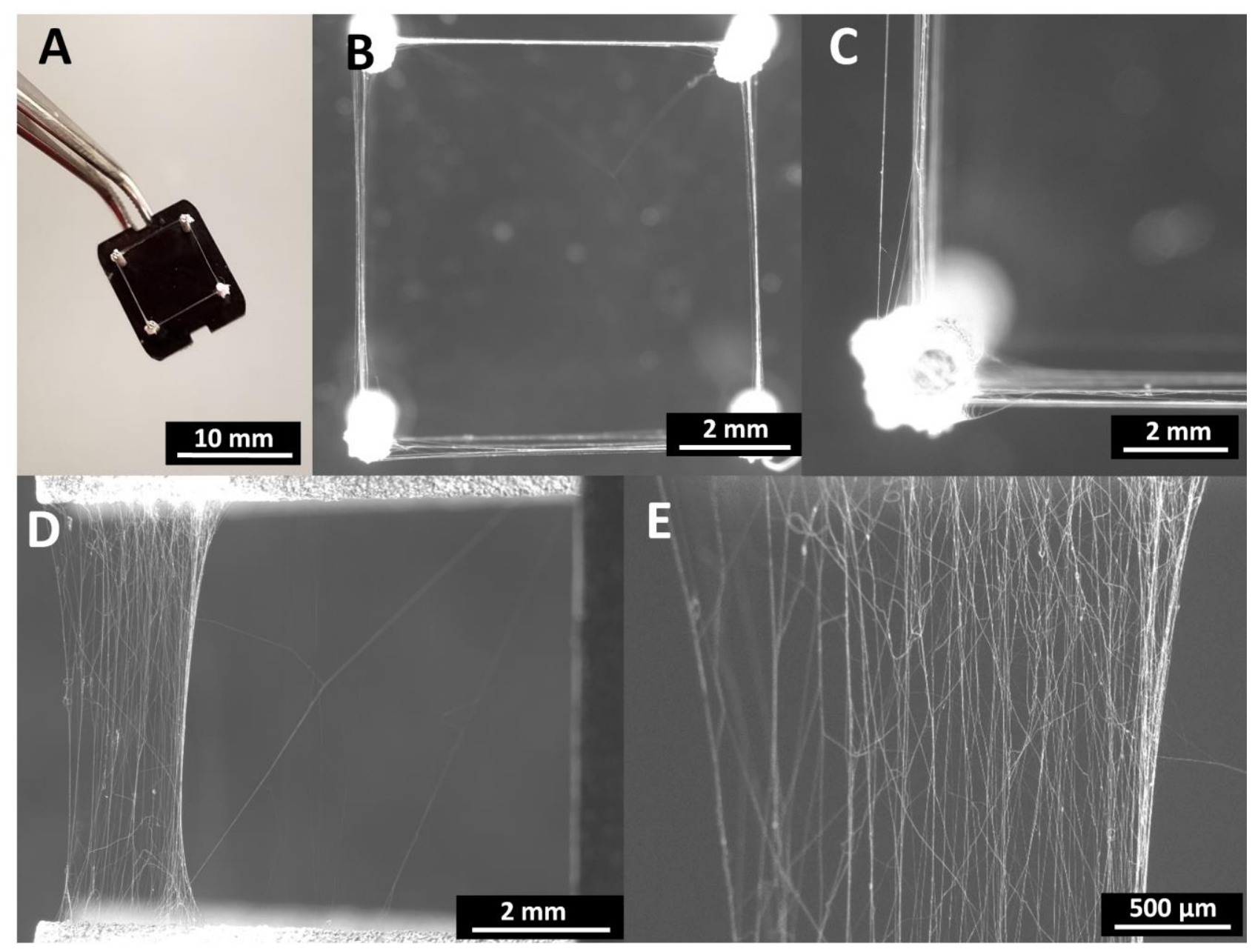

Figure 5. Square pattern generation of suspended nanofiber arrays. (A) Photograph of electrode supporting fibrous arrays. (B) Top down view of the fibrous array. (C) High magnification of the top of an electrode pin. (D) Side view microscopy image of mesh nanofibrous array. (E) Increased magnification of image D.

\subsubsection{Characterizing fiber diameter}

Fiber diameter and morphology was assessed using SEM. An example of a fiber array generated using SI patterning is shown in figure 6 (A). Beneath the fibres is the carbon tape used for fixing the sample, with a pocketed surface. The fiber array is seen to splay from the electrode pillars as well as entangle and overlap. The diameter distribution histogram of this sample in figure 6 (B) shows a typical submicron average diameter reading with mean diameter of ca. $300 \mathrm{~nm}$. Figure 6 (C-E) shows the morphology of the fibers from several samples, exemplifying several conditions of (C) overlap, (D) smooth topography, and (E) entangled. The average diameter of each fiber is indicated showing the effect of entanglement on the overall diameter. Deposition was effective, however there remains many confounding parameters which influence fiber morphology including residual static charge on the fiber, leading to inter-fiber attraction and entanglement. Figure 6 (F) shows the surface of the pillar electrode, with a considerable build-up of nanofibrous mesh layers to the right of the pillar as a consequence of 150 repeated pattern passes at multiple heights along the pillar. 


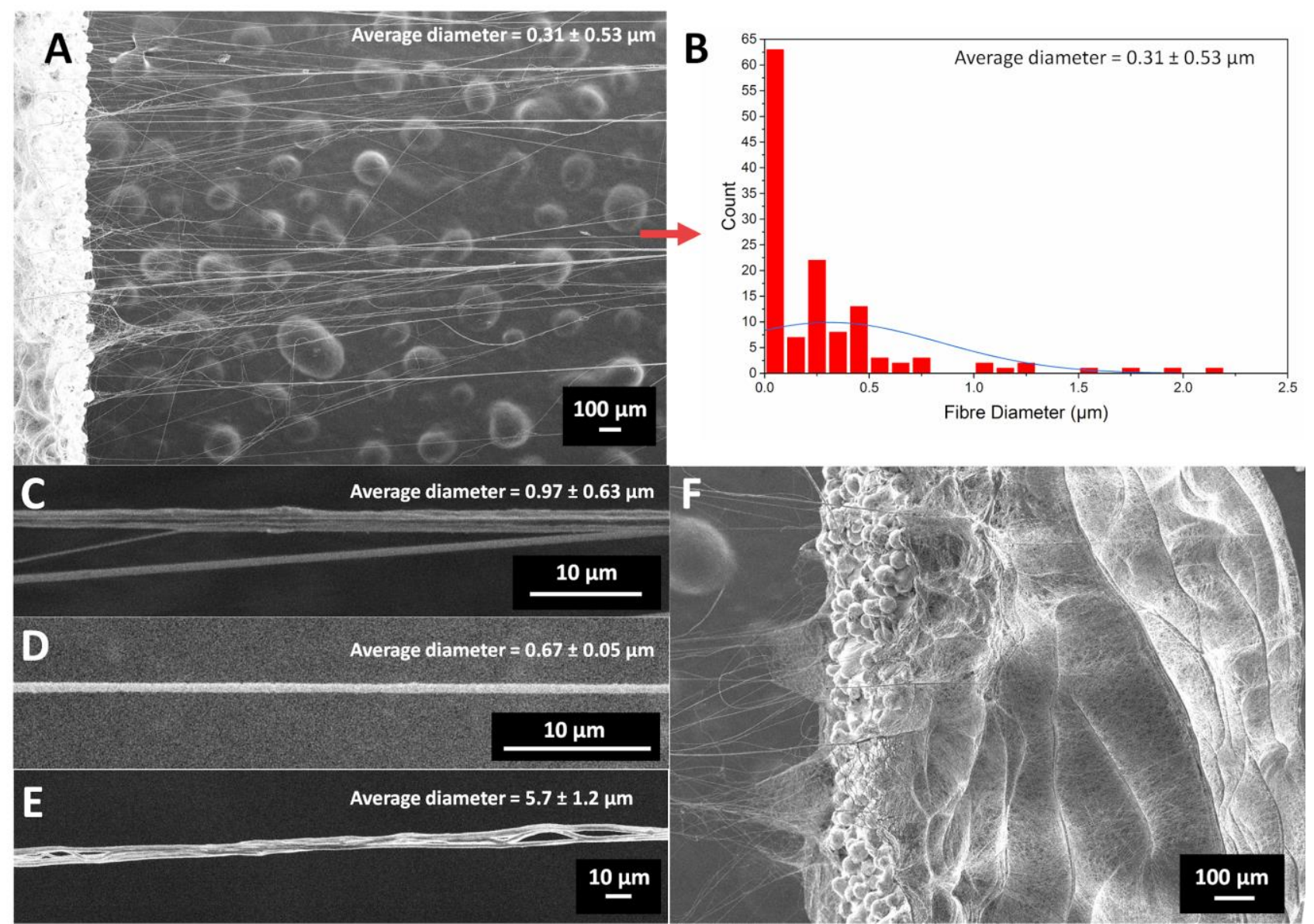

Figure 6. SEM and Diameter distributions. (A) Representative high magnification SEM micrograph of PEO nanofiber mesh, generated using iterated square pattern. (B) Diameter histogram of for sample A. (C-E) High magnification SEM of PEO fibers. (C) Showing crossover intertwining for sample shown in A generated using Square iteration pattern. (D) Example of desirable fiber morphology with average diameter of $0.67 \pm 0.05 \mu \mathrm{m}$ generated using SW pattern. (E) Showing intertwined fibers generating bundle with average diameter of $5.7 \pm 1.2 \mu \mathrm{m}$ generated using SW pattern (F) Morphology of nanofibrous build-up on a single electrode.

\subsubsection{Discussion of Technique and Outlook}

The enclosure and control of humidity had a significant effect on the outcomes of the patterning process. Humidity was found to have a dramatic effect on the success rate of fiber deposition. We define success as: observation of suspended fibers, excluding fibers connected to the electrode base. A $76 \%$ (26 out of 34 samples) success rate was found for a working distance of $1 \mathrm{~mm}$ within an environment with a humidity level between 30-50\% RH, whereas no attempts were successful at higher humidities of $50-62 \% \mathrm{RH}$. This may be caused by a discrepancy in fiber drying rate. In a lower humidity environment (i.e. lower vapour pressure) the drive for water to evaporate is greater than at higher humidity. At high humidity levels fibers would remain partially in solution as they form, leading to discontinuity in jetting as well as failure to support suspension.

The mounting of the syringe pump directly onto the Z-axis reduced issues of dead volume and fluid response delay, which are associated with extended tubing passing any highly viscous polymer solution. This can be particularly important when working with high cost per weight materials, such as collagen, or hexafluoroisopropanol, a common electrospinning solvent. 
NFES technical development has progressed in terms of stage precision, system automation, initiation ${ }^{29}$, high speed camera observation ${ }^{30}$, and through machine vision ${ }^{31,32}$. However, NFES research has rarely focused on electrode geometry, despite this being a relatively common field of investigation in conventional electrospinning research. Suspended 3D fibre mesh macrostructures have been achieved using far-field electrospinning using dumbbell ${ }^{21}$, mandrel ${ }^{33}$, staircase ${ }^{34}$, tracks ${ }^{35}$, and pin array ${ }^{23}$ electrode collectors. These techniques used large working distances, allowing the fibres to whip according to the principle of electrostatic equilibrium. They build layers of fibre on the collector forming macroscopic fibrous meshes, which bow due to the mechanism of deposition. In contrast, the suspension NFES technique achieves control over fibre placement in $\mathrm{Z}$ space via direct inter-pin deposition by working in the near-field domain (below $\sim 10 \mathrm{~mm}$ ).

Suspension NFES has generated nano-arrays for a number of applications ${ }^{22,25,26,36-38}$, including sensors ${ }^{25}$, microfluidics ${ }^{26}$ and flexible electronics ${ }^{3639}$. Bisht et al. demonstrated the precision and accuracy of low voltage NFES by suspending nanofiber across carbon posts with diameter of $30 \mu \mathrm{m}$ and an interspacing of $100 \mu \mathrm{m}^{22}$. However, in each case, the suspension is limited to $2 \mathrm{D}$, i.e. these examples do not produce fibres beyond the platform created by their substrate, working only in a single $\mathrm{Z}$ plane. Liu et al. investigated another example of 3D collector use with NFES, which produced highly aligned $3 \mathrm{D}$ fibres upon a rotating cylindrical collector ${ }^{40}$. However, the pattern itself does not control the structure beyond the curved surface of the collector, i.e. it cannot increase fibre density within the volume of the collector. In comparison, the technique presented here directly controls fibre spatial placement and density, by both the electrode structure and the pattern applied.

The electrodes produced for this study applied a pillar array structure to both support and draw the jetting nanofiber. With these considerations, 4-pillar electrodes could have been produced by traditional manufacturing. However, SLM achieves this production in a single part at a speed far greater than multiple element assembly. SLM allows control over all features of electrode design, reproducibly achieving feature sizes as small as $20 \mu \mathrm{m}$. Titanium was chosen as a robust electrode material that could remain rigid despite this small cross section. The single element electrodes' compact size allowed portability and complete analysis by SEM.

This is the first time such a technology has been applied in NFES methodology. The 4-pillar design keeps pillars at a fixed distance from one another and allows for simple and secure connection. The potential for customisation allows for variance of pin electrode configurations in terms of number, distribution, size and orientation. Future work will introduce multiple electrode design configurations which will take advantage of the rapid prototyping capabilities of SLM, producing more complex electrode structures for use in this suspension NFES technique. This process allows for the exploration of higher complexity patterning techniques using the capabilities of SLM for substrate generation to achieve greater control over fiber array structure and density. As explorations into tissue engineering constructs inexorably become more complex, the scope that SLM offers in terms of customisation and rapid manufacturability can have a positive impact upon research in this area. Initial investigations of its use begins with controlled designs and setups, as explored in this study.

As a device, NFES is relatively contemporary, commonly with fixed functionalities, and with very few commercial devices available ${ }^{41}$. Current commercial systems lacked key features for this study and this necessitated the development of a NFES system in-house to demonstrate a new suspension NFES technique. This was achieved through instrument assembly and through software written in the high speed language $\mathrm{C}++$, allowing instruments to work in concert following g-code script. The g-code writing program allowed for calibration of each pillar electrode, and each initialising pin. This was done 
by translating pattern values and calibration inputs into G-code sequences with interspersed cleaning routines. This allows for semi-automatic patterning, which can operate for any length of time, and once initiated it can be made more accurate with calibration routines to accommodate minor electrode differences.

The ultimate use of these suspended nanoarrays is within engineered microtissue constructs. Tissue engineering depends upon 3D architectures, and encapsulation of 3D fibrous structures has been successful in achieving a positive cellular response in many cases ${ }^{42-44}$. Future work will investigate the encapsulation of these fibrous arrays in gel systems such as collagen and synthetic hydrogels to achieve the interconnected porosity as well as the fibrous network structure of the ECM. Greater volume nanoarray fabrication is expected to resolve the challenges of producing hierarchical tissue constructs in 3D. The findings of this study are significant as it creates, for the first time, a suspended nanoarray in a directed manner which can be extended across multiple working planes in situ. This work highlights the potential of this new approach as a means of developing 3D scaffold nanostructures large enough for use in microtissue constructs for the medical research sector.

\section{Conclusions}

There remain challenges in redefining fabrication techniques to achieve complex 3D, hierarchical structures, while retaining nanoscale features. The layer-by-layer NFES method has several limitations when addressing these challenges, and so a suspension-based NFES technique was developed. A semiautomated NFES system was purpose built and used to electrospin onto (a) planar silicon and (b) 3D titanium electrodes. The suspended nanofibrous arrays produced were analysed for their orientation distribution and diameter. Microscopy showed orientation of fibers to align with patterning toolpaths, which achieved an average fiber diameters below one micron.

This established the principles of this technique in demonstrating the directed 3D suspension of nanofibers. A major goal of this technique was the direct patterning in an accurate and reproducible manner. Construction of consistent nanofibrous arrays will facilitate future cell culture studies examining the effects of nanofiber arrays distribution in 3D. To achieve greater precision, further optimisation of this new technique is required, in terms of parameter and electrode variation studies. With this 3D nanofabrication technique and SLM manufactured electrodes, novel high volume nanofibrous architectures can be generated, bridging the gap of additive nanofabrication. While this study explored initial geometries, it is a necessary first step towards the exploration of complex topologies that can pave the way for generating higher order systems to match the demands of tissue engineering.

\section{Acknowledgements}

The authors acknowledge the financial support of the Australian Research Council (ARC) Centre of Excellence Scheme (Project CE 140100012). The ARC had no involvement in the studies design; in the collection, analysis or interpretation of data; in the writing of the report; or in the decision to submit the article for publication. The authors make no declarations of interest. The authors thank the Australian National Fabrication Facility (ANFF) for providing facilities for the fabrication of the NFES system components. The authors thank the University of Wollongong Electron Microscopy Centre (EMC) for providing SEM facilities. The authors thank Mr. Grant Barnsley for his support in the fabrication of collector electrode arrays, and thank Mr. Benjamin Filippi for his assistance in device manufacturing. 


\section{References}

1 V. Beachley and X. Wen, Prog. Polym. Sci., 2010, 35, 868-892.

2 F. Yang, R. Murugan, S. Wang and S. Ramakrishna, Biomaterials, 2005, 26, 2603-2610.

3 E. Kijeńska, M. P. Prabhakaran, W. Swieszkowski, K. J. Kurzydlowski and S. Ramakrishna, Eur. Polym. J., 2014, 50, 30-38.

4 J.-H. He, Y. Liu and L. Xu, Mater. Sci. Technol., 2010, 26, 1275-1287.

5 W. S. Khan, R. Asmatulu, M. Ceylan and A. Jabbarnia, Fibers Polym., 2013, 14, 1235-1247.

6 W.-E. Teo, R. Inai and S. Ramakrishna, Sci. Technol. Adv. Mater., 2011, 12, 013002.

7 Y. Huang, Y. Duan, Y. Ding, N. Bu, Y. Pan, N. Lu and Z. Yin, Sci. Rep., 2014, 4, 5949.

8 F.-L. He, D.-W. Li, J. He, Y.-Y. Liu, F. Ahmad, Y.-L. Liu, X. Deng, Y.-J. Ye and D.-C. Yin, Mater. Sci. Eng. C Mater. Biol. Appl., 2018, 86, 18-27.

9 J. He, F. Xu, R. Dong, B. Guo and D. Li, Biofabrication, 2017, 9, 015007.

10 G. Hochleitner, T. Jüngst, T. D. Brown, K. Hahn, C. Moseke, F. Jakob, P. D. Dalton and J. Groll, Biofabrication, 2015, 7, 035002.

11 M. Lee and H.-Y. Kim, Langmuir, 2014, 30, 1210-1214.

12 G. Luo, K. S. Teh, Y. Liu, X. Zang, Z. Wen and L. Lin, ACS Appl. Mater. Interfaces, 2015, 7, $27765-$ 27770.

13 G. Luo, K. S. Teh, X. Zang, D. Wu, Z. Wen and L. Lin, in 2016 IEEE 29th International Conference on Micro Electro Mechanical Systems (MEMS), 2016.

14 J. Visser, F. P. W. Melchels, J. E. Jeon, E. M. van Bussel, L. S. Kimpton, H. M. Byrne, W. J. A. Dhert, P. D. Dalton, D. W. Hutmacher and J. Malda, Nat. Commun., 2015, 6, 6933.

15 G. Hochleitner, T. Jüngst, T. D. Brown, K. Hahn, C. Moseke, F. Jakob, P. D. Dalton and J. Groll, Biofabrication, 2015, 7, 035002.

16 J. Visser, F. P. W. Melchels, J. E. Jeon, E. M. van Bussel, L. S. Kimpton, H. M. Byrne, W. J. A. Dhert, P. D. Dalton, D. W. Hutmacher and J. Malda, Nat. Commun., 2015, 6, 6933.

17 Y.-K. Fuh, Y.-C. Wu, Z.-Y. He, Z.-M. Huang and W.-W. Hu, Mater. Sci. Eng. C Mater. Biol. Appl., 2016, 62, 879-887.

18 P. Fattahi, J. T. Dover and J. L. Brown, Adv. Healthc. Mater., , DOI:10.1002/adhm. 201700456.

19 A. Abbott, Nature, 2003, 424, 870-872.

20 W. E. Teo and S. Ramakrishna, Nanotechnology, 2005, 16, 1878-1884.

21 P. Zhu, A. Lin, X. Tang, X. Lu, J. Zheng, G. Zheng and T. Lei, AIP Adv., 2016, 6, 055304.

22 G. S. Bisht, G. Canton, A. Mirsepassi, L. Kulinsky, S. Oh, D. Dunn-Rankin and M. J. Madou, Nano Lett., 2011, 11, 1831-1837.

23 K. Zhang, X. Wang, D. Jing, Y. Yang and M. Zhu, Biomed. Mater., 2009, 4, 035004.

24 D. Li, Y. Wang and Y. Xia, Adv. Mater., 2004, 16, 361-366.

25 D. Chen, X. Guo, Z. Wang, P. Wang, Y. Chen and L. Lin, in 2011 IEEE 24th International Conference on Micro Electro Mechanical Systems, 2011.

26 SangHoon Lee, S. Lee, K. Limkrailassiri, Y. Gao, C. Chang and L. Lin, in 2007 IEEE 20th International Conference on Micro Electro Mechanical Systems (MEMS), 2007.

27 M. Lee and H.-Y. Kim, Langmuir, 2014, 30, 1210-1214.

28 Y. Huang, Y. Duan, Y. Ding, N. Bu, Y. Pan, N. Lu and Z. Yin, Sci. Rep., 2014, 4, 5949.

29 G. Bisht, S. Nesterenko, L. Kulinsky and M. Madou, J. Lab. Autom., 2012, 17, 302-308.

30 W. Yang, H. Duan, C. Li and W. Deng, Phys. Rev. Lett., 2014, 112, 054501.

31 K.-S. Kwon and D.-Y. Lee, J. Micromech. Microeng., 2013, 23, 065018.

32 T. H. Phung, S. Kim and K.-S. Kwon, J. Micromech. Microeng., 2017, 27, 095003.

33 F. Liang, F. Fang, J. Zeng, Z. Wang, W. Ou, X. Chen, P. Wu, H. Wang and L. Zhang, AIP Adv., 2017, 7, 025002.

34 A. Kriebel, M. Rumman, M. Scheld, D. Hodde, G. Brook and J. Mey, J. Biomed. Mater. Res. B Appl. Biomater., 2014, 102, 356-365.

35 V. Beachley, E. Katsanevakis, N. Zhang and X. Wen, Adv. Healthc. Mater., 2012, 2, 343-351.

36 Y.-K. Fuh, S.-Y. Chen and J.-C. Ye, Appl. Phys. Lett., 2013, 103, 033114.

37 J. Pu, X. Yan, Y. Jiang, C. Chang and L. Lin, Sens. Actuators A Phys., 2010, 164, 131-136.

38 C. Chang, V. H. Tran, J. Wang, Y.-K. Fuh and L. Lin, Nano Lett., 2010, 10, 726-731.

39 C. Shen, C.-P. Wang, M. Sanghadasa and L. Lin, RSC Adv., 2017, 7, 11724-11731. 
40 Z. H. Liu, C. T. Pan, C. K. Yen, L. W. Lin, J. C. Huang and C. A. Ke, Appl. Surf. Sci., 2015, 346, $291-$ 301.

41 X.-X. He, J. Zheng, G.-F. Yu, M.-H. You, M. Yu, X. Ning and Y.-Z. Long, J. Phys. Chem. C, 2017, 121, $8663-8678$.

42 I.-C. Liao, F. T. Moutos, B. T. Estes, X. Zhao and F. Guilak, Adv. Funct. Mater., 2013, 23, 5833-5839.

43 L. A. Bosworth, L.-A. Turner and S. H. Cartmell, Nanomedicine, 2013, 9, 322-335.

44 R. E. McMahon, X. Qu, A. C. Jimenez-Vergara, C. A. Bashur, S. A. Guelcher, A. S. Goldstein and M. S. Hahn, Tissue Eng. Part C Methods, 2011, 17, 451-461. 


\section{Supplementary}

\section{Section S1.}

The ACD was comprised of an acrylic bed holding two servo motors rotating two paddles. These paddles were covered in polyester sponge and supported by circular bearings. When the Z-stage is lowered to a certain height it triggers an electronic switch, mounted on the stage. The switch initiates a servo driven compression motion of the paddles around the emitter tip. The tip is then retracted and cleaned by the drawing motion of the sponge. The ACD then resets the servos and moves the bed $1 \mathrm{~mm}$ in the Y direction. This allows an uncontaminated region of the paddles to be used in the following clean procedure. This $1 \mathrm{~mm}$ move is achieved by signalling a stepper motor to rotate a gear attached to a rack and pinion embedded in the ACD bed. The servos, switch and stepper motor were controlled using a microcontroller board (Arduino Mega 2560) connected to a PCB (Ultimaker v1.5.7).
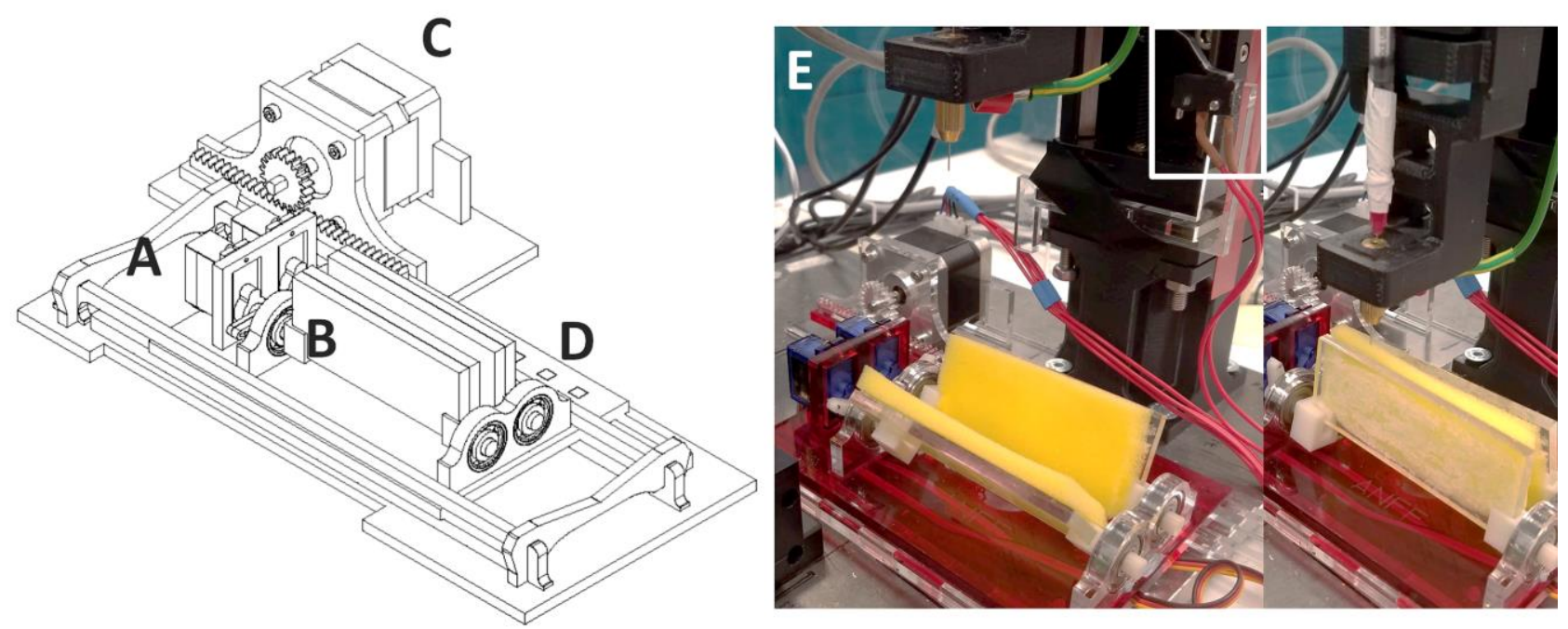

Figure S1. Automatic Cleaning Device. (A) Servos, (B) Paddles, (C) Stepper motor, (D) Bearings. (E) Three part cleaning routine, with switch mounted on the $Z$ axis highlighted. 


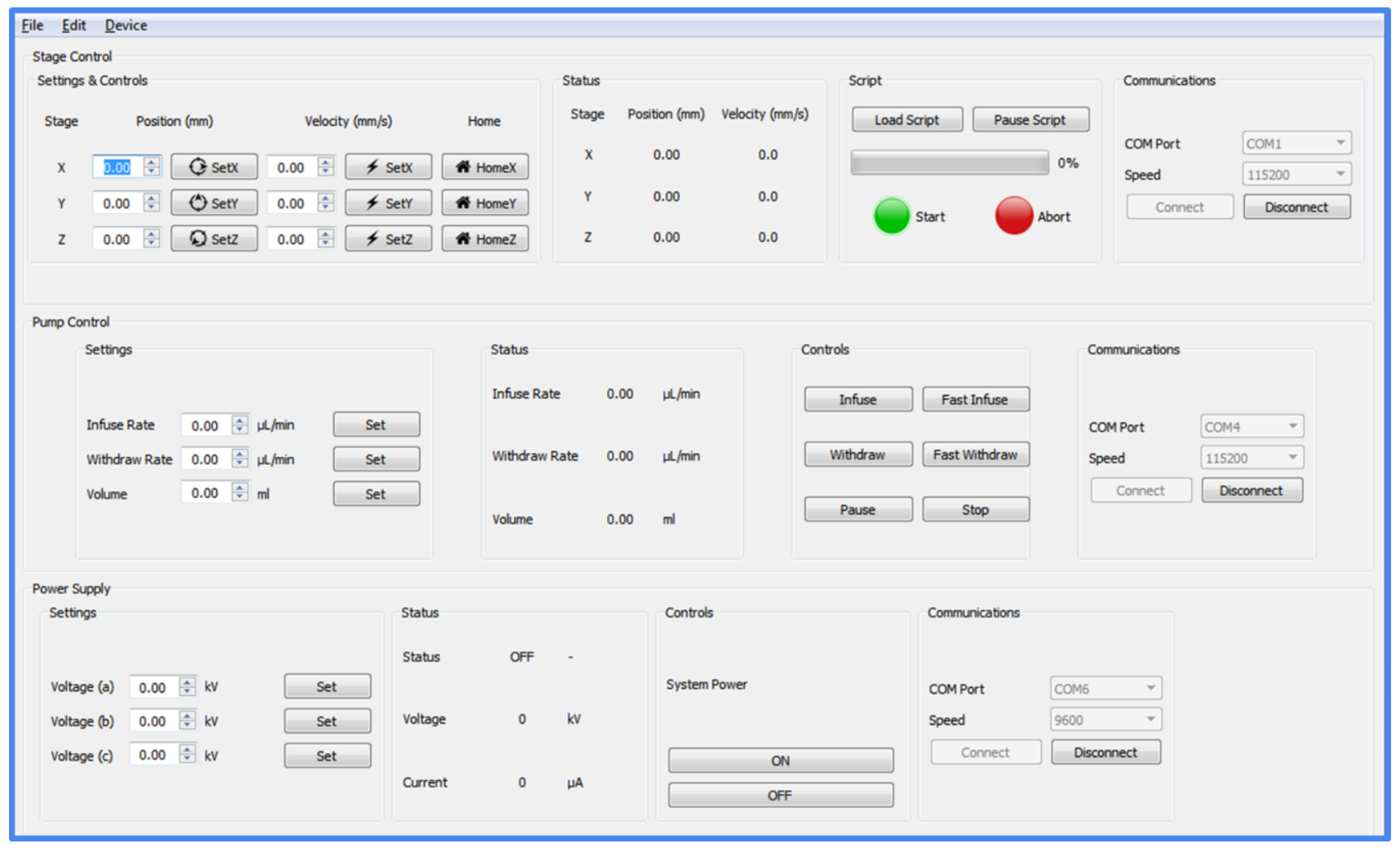

Figure S2. Graphic User Interface.

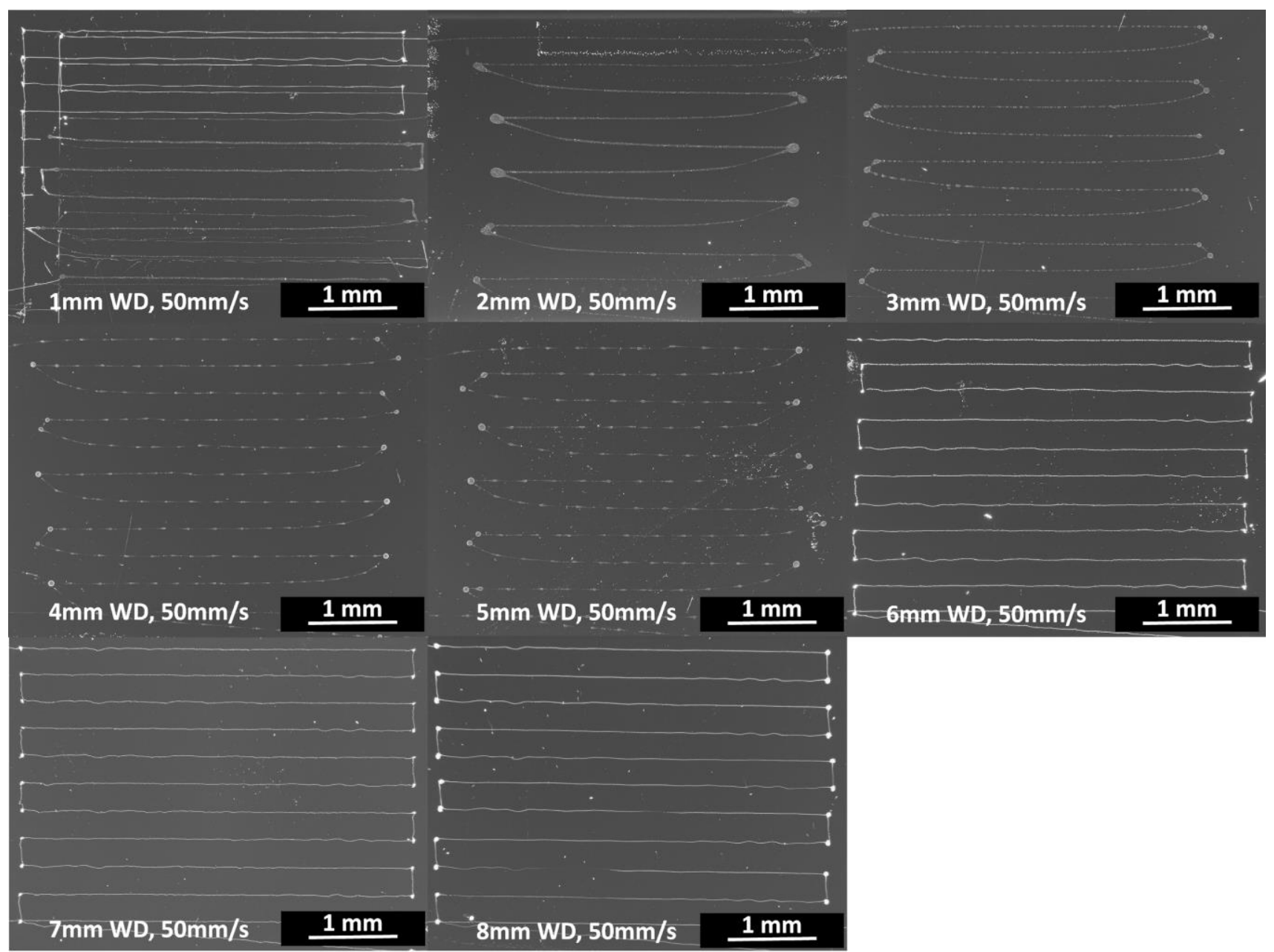


Figure S3. Microscopy images of 2D patterned nanofibres on silicon substrate using varied working distances.

Supplementary video 1 . Square wave pattern operation.

Supplementary video 2. Square iteration pattern operation.

Supplementary video 3. Demonstration of patterning procedure from high magnification camera perspective. 